\title{
Active deformation fields: Dense deformation field estimation for atlas-based segmentation using the active contour framework
}

\author{
Subrahmanyam Gorthi ${ }^{\mathrm{a}, *, 1}$, Valérie Duay ${ }^{\mathrm{b}, 1}$, Xavier Bresson ${ }^{\mathrm{c}}$, Meritxell Bach Cuadra ${ }^{\mathrm{a}}$, \\ F. Javier Sánchez Castro ${ }^{\mathrm{d}}$, Claudio Pollo ${ }^{\mathrm{a}, \mathrm{e}}$, Abdelkarim S. Allal ${ }^{\mathrm{f}}$, Jean-Philippe Thiran ${ }^{\mathrm{a}}$ \\ a Signal Processing Laboratory (LTS5), Ecole Polytechnique Fédérale de Lausanne (EPFL), Switzerland \\ ${ }^{\mathrm{b}}$ Institute of Bio-Engineering, University of Applied Sciences Western Switzerland (HES-SO Genève), Geneva, Switzerland \\ ${ }^{\mathrm{c}}$ Department of Mathematics, UCLA, Los Angeles, USA \\ ${ }^{\mathrm{d}}$ Medisys Research Lab., Philips Healthcare, Suresnes, France \\ e Department of Neurosurgery, Centre Hospitalier Universitaire Vaudois (CHUV), Lausanne, Switzerland \\ ${ }^{\mathrm{f}}$ Service de Radio-oncologie, Hôpital Fribourgeois, Fribourg, Switzerland
}

\section{A R T I C L E I N F O}

\section{Article history:}

Received 27 August 2010

Received in revised form 15 March 2011

Accepted 11 May 2011

Available online 23 May 2011

\section{Keywords:}

Atlas-based segmentation

Joint registration and segmentation model

Hierarchical atlas registration

Active contours

Level set representation

\begin{abstract}
A B S T R A C T
This paper presents a new and original variational framework for atlas-based segmentation. The proposed framework integrates both the active contour framework, and the dense deformation fields of optical flow framework. This framework is quite general and encompasses many of the state-of-the-art atlas-based segmentation methods. It also allows to perform the registration of atlas and target images based on only selected structures of interest. The versatility and potentiality of the proposed framework are demonstrated by presenting three diverse applications: In the first application, we show how the proposed framework can be used to simulate the growth of inconsistent structures like a tumor in an atlas. In the second application, we estimate the position of nonvisible brain structures based on the surrounding structures and validate the results by comparing with other methods. In the final application, we present the segmentation of lymph nodes in the Head and Neck CT images, and demonstrate how multiple registration forces can be used in this framework in an hierarchical manner.
\end{abstract}

(C) 2011 Elsevier B.V. All rights reserved.

\section{Introduction}

Atlas-based segmentation is a widely used technique for automated segmentation of medical images (see Rohlfing et al. (2005) for a survey). It relies on the existence of a reference image (called atlas) in which structures of interest have been accurately segmented, usually by hand. To segment a new image (called target image), a dense deformation field that puts the atlas into a pointto-point spatial correspondence with the target image is first computed. This transformation is then used to project label maps assigned to structures from the atlas onto the target image to be segmented. This way, the segmentation problem is reduced to a registration problem that tries to capture and compensate the normal anatomical variabilities.

The main advantage of the atlas-based segmentation method is that the dense deformation field, interpolated on the whole image

\footnotetext{
* Corresponding author.

E-mail addresses: subrahmanyam.gorthi@epfl.ch (S. Gorthi), valerie.duay@ hesge.ch (V. Duay), xbresson@math.ucla.edu (X. Bresson), meritxell.bach@epfl.ch (M.B. Cuadra), javier.sanchez-castro@philips.com (F.J. Sánchez Castro), Claudio Pollo@chuv.ch (C. Pollo), allala@h-fr.ch (A.S. Allal), JP.Thiran@epfl.ch (J.-P. Thiran).

1 Both authors contributed equally to this work.
}

from the registration of visible image features, allows to easily estimate, in the target image, the position of structures with fuzzy or no visible contours. Moreover, this approach allows to segment at the same time several contours of any types (closed, open, connected or disconnected) provided that they have been defined in the atlas image. The accuracy of the segmentation results depend on the segmentation of the reference image and mainly on the quality of the registration between the reference image (the atlas) and the target image. A wide range of image registration techniques that allow to deform a given atlas to a subject have been developed over the last 20 years (Bajcsy and Kovacic, 1989; Christensen et al., 1994; Rueckert et al., 1999; Thirion, 1998). To compute the deformation field, they generally optimize some global similarity measure (such as mutual information, sum of squared differences or cross-correlation) coming from the atlas intensity image.

The main limitation of the image registration methods commonly used for atlas registration is that they often lead to a compromise between the accuracy of the registration and the smoothness of the deformation. When at some places the registration is not accurate enough, a widely-used solution is to globally or locally allow more variability in the registration model in order to obtain more local deformation, but with the risk of creating 
irregularities in the deformation field (Duay et al., 2004). Also, this does not assure that the desired level of precision will be obtained. Another limitation of these methods is that they assume that a point to point correspondence exists between the atlas and the images to be segmented. Such assumption can lead to an inaccurate registration, particularly, in the presence of content-based inconsistencies between the atlas and the target image. ${ }^{2}$

In this paper, we propose a general framework that encompasses many of the existing complementary registration methods. Thus, it facilitates to combine the advantages of different registration approaches. It can perform both the registration and segmentation tasks jointly. We also propose a new label function representation that can represent multiple labels using a single level set function, and it enables to perform the registration based on only the selected consistent structures of interest in atlas and target images.

The rest of the paper is organized as follows. Section 2 presents a brief review of the state-of-the-art methods that integrate the AC framework in the registration process. Section 3 presents the main contributions of this paper that include general formulation of our active deformation fields model and a new multi-phase active contour representation. Section 4 presents various types of registration/segmentation forces that can be used in our framework, comparison of our new multi-phase representation with two other state-of-the-art methods that can be derived from our general framework, regularization constraints, and an hierarchical approach that we use for applying registration forces. In Section 5, we present three clinical applications. Finally, conclusions are presented in Section 6.

\section{State-of-the-art: Joint registration and active contour segmentation}

This is not the first attempt to combine registration and active contour (AC) segmentation. Concerning the algorithms deduced from the level set evolution equation, we cite as the first reference, the "morphing AC model" proposed by Bertalmío et al. (2000), despite the fact that this method combines morphing instead of registration to AC segmentation. This model deforms the moving image to the target image as in a registration process but the corresponding geometric transformation is not determined explicitly. The goal is to identify in the target image, a contour that corresponds to the object segmented in the reference image. This is done by solving a system of two partial differential equations (PDEs). The first one is in charge of morphing the two images. The second one is a tracking equation that makes the level set function evolve with the same velocity given by the morphing equation. Vemuri et al. (2003) have proposed an algorithm very close to the "morphing AC model" of Bertalmío, but dedicated for atlas registration. The main difference is that the image matching is not anymore tracked by a level set function but by a deformation field and that the morphing and the tracking PDEs are combined in a single PDE. To get the segmentation of a particular object in the target image, the computed deformation is applied to the segmentation of the corresponding object in the moving image following the principles of the atlas-based segmentation method. This model is further analyzed in more detail in Section 3.1.

The first attempt to model the atlas-based segmentation directly from the energy equations is made by Yezzi et al. (2001). This model first defines two segmentation energies, one in the moving image and another one in the target image, that aim to seg-

\footnotetext{
2 The term "content-based inconsistencies" in this paper refers to the presence of certain structures (for example, a tumor), either in the atlas, or in the target image, but not in both. The example presented in Section 5.2 further illustrates such contentbased inconsistencies.
}

ment the same object in both the images. Then both energies are coupled by defining the active contour of the target image as being the active contour of the moving image under a particular deformation. The advantage of this method is that it can combine multi-modal information since the segmentation energies are defined independently on both the images.

Inspired by the Yezzi's model, several authors have proposed other joint registration and AC segmentation models. Moelich and Chan (2003) have shown that Yezzi's model is very sensitive to the initial active contour position and deformation. To avoid local minima, the active contours of both images have to be initially already well superposed to the objects of interest. Thus they have proposed to define the active contour of both images (not only of the target image as in the Yezzi's model) as being the initial curve under a particular deformation. They have also introduced a preregistration step to find out the best initial registration parameters that compensate in both images the difference of positions between this initial curve and the objects of interest. Unal and Slabaugh (2005) have generalized the rigid framework of Yezzi to non-rigid registration. An et al. (2005) have further included a prior segmentation term in their model that computes the intensity difference between the prior shape and the shape to be segmented by the active contour in the target image. This term in fact corresponds to the matching image term used in the Bertalmío and Vemuri's models with the difference that it is computed only inside the prior shape. Young and Levy (2005) have proposed to refine in a second step, the segmentation result obtained by the Yezzi's model with the "morphing AC model" of Bertalmío. Finally, (Droske et al., 2009) have presented two joint registration and AC segmentation models. The first model is similar to Yezzi's model, but optimizes a non-rigid deformation that is then propagated from the contour to the whole image. Their second model did not use anymore a level set function to represent the contour. It is based on an energy that optimizes the detection of common contours between the source and target images. Thus close, open, connected and disconnected contours can be considered for the registration. However, this model is limited to contour-based registration, and cannot exploit region-based features.

Other types of models close to this joint registration and active contour segmentation framework have been proposed. First, there are algorithms derived from joint optical flow registration and active contour segmentation that aim to generate a dense but "discontinuous" deformation field on the whole image for the study of motion in image sequences (Amiaz and Kiryati, 2006; Paragios et al., 2002). Also Paragios et al. (2003) have extracted the non-rigid deformation between 2D geometric shapes by representing them with a level set function. This last model extracts the deformation only on the active contours.

The main contribution of this paper is a new framework that combines the advantages of the models described above and overcomes some of their limitations. First, as in An et al. (2005), we consider the moving image as a prior image (the atlas). The initial shapes of the active contours are given by the objects of interest that are manually delineated in this prior image. Then, as in Moelich and Chan (2003), we perform an initial global registration step in order to compensate for the initial differences of position between the atlas and the target image. Also, as in Droske et al. (2009), our algorithm computes a dense non-rigid deformation field on the whole image. Similar to Vemuri et al. (2003), our method has been inspired first by the general evolution equation of the level set function. One of the main differences is that in our scheme, we propose to model several connected and/or disconnected active contours with a new label function representation. This label function permits in particular to consider different segmentation/registration forces at different areas of the image. Inspired by An et al. (2005) and Young and Levy (2005), we propose 
an approach to combine the object-based forces coming from the AC segmentation technique with the pixel-based forces used in Vemuri's model, AC segmentation models with shape prior (Chen et al., 2002), or in optical flow registration algorithms (Barron et al., 1994).

\section{Our active contour-based atlas registration framework}

This section contains the main contributions of this paper, and is organized as follows: The details of related previous work are presented in Section 3.1. General formulation of our active deformation fields model is presented in Section 3.2. A new multi-phase active contour representation is proposed in Section 3.3.

\subsection{Related work}

The main source of inspiration for our joint registration and segmentation algorithm is the partial differential equation (PDE)based model proposed by Vemuri et al. (2003). The formulation of Vemuri's model has been intuitively deduced from the following general level set evolution equation introduced by Osher and Sethian (1988):

$\frac{\partial \phi_{D}(x, t)}{\partial t}=v\left(\phi_{D}(x, t)\right)\left|\nabla \phi_{D}(x, t)\right|$,

where $v$ is the velocity of the flow or speed function that contains the local segmentation and contour regularization constraints, and $\phi_{D}: \Omega \rightarrow \mathbb{R}$ (for the image domain $\Omega$ ) is the signed distance function often used to represent implicitly the active contour by its zero level. The original idea brought by Vemuri's model is to replace in (1), $\phi_{D}$ by the intensity function of the image to register $\phi_{I}: \Omega \rightarrow \mathbb{R}$ (the moving image). This leads to the following equation:

$\frac{\partial \phi_{I}(x, t)}{\partial t}=v\left(\phi_{I}(x, t)\right)\left|\nabla G_{\sigma} * \phi_{I}(x, t)\right|$,

where $G_{\sigma}$ is a Gaussian kernel with standard deviation of $\sigma$, and $*$ is the convolution operator. The image $\phi_{I}$ is prior smoothed with a Gaussian filter because the gradient computation is very sensitive to noise. The level sets considered in the segmentation process correspond to the contours naturally present in the moving image, i.e., the curves of high image gradient. The speed function used in both Bertalmío's and Vemuri's model is the intensity difference term $v\left(\phi_{I}(x, t)\right)=\left(\phi_{I}(x, t)-\phi_{T}(x)\right)$, where $\phi_{T}(x)$ is the intensity function of the target image.

Note that Eq. (2) gives only the intensity evolution, but not the geometric transformation between the images. So, in order to explicitly track the deformation field, they have intuitively derived one more equation presented below. The deformation field vector $u: \mathbb{R}^{n} \rightarrow \mathbb{R}^{n}$ (typically, $n=\{2,3\}$ ) is given by:

$\frac{\partial u(x, t)}{\partial t}=\left(\phi_{I}(x, t)-\phi_{T}(x)\right) \frac{\nabla G_{\sigma} * \phi_{I}(x, t)}{\left|\nabla G_{\sigma} * \phi_{I}(x, t)\right|_{\epsilon}}$,

where $|\Gamma|_{\epsilon}=\sqrt{|\Gamma|^{2}+\epsilon^{2}}$, and $\epsilon$ is a small positive constant used for avoiding the singularity.

The intensity function $\phi_{I}$ at time $t$ is given by the deformation field $u(x, t)$ and the initial intensity function $\phi_{I}(x, 0)$ such that:

$\phi_{I}(x, t)=\phi_{I}(x+u(x, t), 0)$,

which ensures that the evolution of the intensity function exactly corresponds to the current deformation.

The deformation forces of Vemuri's model are pixel-based forces computed on the whole image. This has two main consequences. First, this algorithm presents some limitations when it has to recover global differences because the registration forces that it uses are very local. Secondly, it fails to register regions when texture is different with similar intensity distributions. This situation could, for example, arise in natural images (Paragios and Deriche, 2002) in which two regions (object and background in this example) have similar intensity distributions, but different textures. This can happen in medical images also, particularly, in the segmentation of ultrasound images (Hui et al., 2009). Finally, Vemuri's model has no scheme to prevent the registration of inconsistent regions.

\subsection{Our model: Active deformation fields}

The general formulation of our model is based on tracking of the level set function motion based on the conservation of morphological (i.e., shape) description. Since level sets are basically a morphological notion, the conservation of morphological description essentially states that level set function is preserved under small deformation field and a short period of time. Let " $\phi_{G}$ " represent a general level set function; it means that wherever $\phi_{G}$ is used, it is applicable to different types of level set functions like signed distance function $\left(\phi_{D}\right)$, intensity function $\left(\phi_{I}\right)$, or the label function $\left(\phi_{L}\right)$ that will be introduced in Section 3.3. Now, the above mentioned conservation implies that $\phi_{G}$ remains constant for small displacements and a short period of time. This results in the following equation:

$\phi_{G}(x, t)=\phi_{G}(x+d u, t+d t) \Rightarrow d \phi_{G}(x, t)=0$,

where $d \phi_{G}$ is the total derivative of $\phi_{G}$. By using the chain rule, this constraint can be rewritten as the evolution equation of a vector flow:

$\frac{\partial u(x, t)}{\partial t}=-\frac{\phi_{G, t}}{\left|\nabla \phi_{G}\right|} N_{\phi_{G}}$

where $\phi_{G, t}$ is given by (1) (with a change of subscript), and it represents the variation of the level set function according to the desired forces such as supervised segmentation, shape prior knowledge or contour regularization; $N_{\phi_{G}}$ in the above equation represents the normal of level set, and is given by:

$N_{\phi_{G}}= \begin{cases}\frac{\nabla \phi_{G}}{\left|\nabla \phi_{G}\right|}, & \text { if } \nabla \phi_{G} \neq 0 \\ 0, & \text { otherwise. }\end{cases}$

Note that the widely used morphological descriptor in registration problems is the "luminance function". With the luminance function, the above mentioned conservation is equivalent to optical flow (OF) assumption, which states that the brightness of the moving image stays constant for small displacements, over short periods of time. In that sense, in our discussions, we will be referring to "conservation of morphological description" simply as "OF approach".

By introducing the evolution equation of the level set segmentation model (1) in (6), we obtain the following formula merging the active contour segmentation framework with the image registration task:

$\frac{\partial u(x, t)}{\partial t}=-v\left(\phi_{G}(x, t)\right) N_{\phi_{G}}$.

The level set function $\phi_{G}$ does not evolve with the usual finite difference scheme. Its position at time $t$ is given by the deformation field $u(x, t)$ and the initial level set function $\phi_{G}(x, 0)$ such that:

$\phi_{G}(x, t):=\phi_{G}(x+u(x, t), 0)$,

where $\phi_{G}(x, 0)$ is the initial active contour position. This ensures that the evolution of the level set function exactly corresponds to the current deformation. Notice that when intensity function is used as the level set function (i.e., when $\phi_{G}=\phi_{I}$ ), the general evolution Eq. (8) is indeed equivalent to gradient flows, where each voxel warps along its intensity-gradient, and its corresponding deformation field is taken into account by (9). Introducing (9) in (8) yields: 
$\frac{\partial u(x, t)}{\partial t}=-v\left(\phi_{G}(x+u(x, t), 0)\right) N_{\phi_{G}}$.

This equation defines a displacement vector at each point of the level set function. The level set function models the contours of the objects selected in the atlas to drive its registration.

As mentioned earlier, different types of level set functions can be used in place of $\phi_{G}$ in the above formulation. For instance, signed distance function $\left(\phi_{D}\right)$ representation can be used; however, the main drawback of $\phi_{D}$ representation is that it can model only two regions. Multi-phase representations have been proposed by combining several signed distance function (see for instance Vese and Chan, 2002; Zhao et al., 1996), but such solution will drastically increase the computational complexity of this model. On the other hand, while the representations like intensity function $\left(\phi_{I}\right)$ can model any type of contours, they are limited to only pixel-based registration forces (see Section 4.1 for more details on $\phi_{D}$ and $\left.\phi_{I}\right)$.

To cope with these limitations, in this paper, we propose a new label function representation of the level sets that can model any number of regions as well as represent various types of registration forces, using a single function, and this is an important contribution of this paper. This new multi-phase active contour representation is described in detail in the following section. ${ }^{3}$

\subsection{New multi-phase active contours representation}

For the registration of multiple regions, we propose a new label function $\phi_{L}$ to represent the active contours selected in the atlas. In the $\phi_{L}$ representation, the contours do not anymore correspond to the zero level set, but to the discontinuities of piecewise constant level set function. This label function permits to define an arbitrary number of regions as follows:

$\phi_{L}: x \in \Omega_{k} \rightarrow \phi_{L}(x)=k, \quad k \in[1, \ldots, n]$ if $x \in \Omega_{k}$,

where $\Omega_{k}$ is the $k$ th labeled region and $n$ is the number of regions. In this representation, active contours are modeled by the discontinuities of $\phi_{L}$.

The main advantage of this label function representation is that it can distinguish any number of regions with a single function. The implementation of this representation is an important challenge; when multiple regions are represented using $\phi_{L}$, it does not contain the polarity information that is essential to compute the widely used region-based forces of the $\mathrm{AC}$ segmentation. Note that the term polarity in this context is defined as the information of direction that indicates the inside $\left(\Omega_{\text {in }}\right)$ and the outside $\left(\Omega_{\text {out }}\right)$ for each modeled region. The importance of polarity information will be more clear with the description of region-based forces in Section 4.5. We now present in detail on how we handle this issue of polarity information.

In order to accurately represent the polarity information for each region, we introduce in the general formulation of our model (10), a sign function: $S\left(\phi_{L}(x)\right)$. The purpose of this sign function is to manipulate the overall direction of the evolution such that it is always oriented in the direction of $\Omega_{\text {in }}$ to $\Omega_{\text {out }}$, independent of the orientation of the gradient of the label function $\phi_{L}(x)$ (which is always from the low intensity label to the high intensity label). In other words, irrespective of the direction of $\nabla \phi_{L}(x)$, the direction of $\left(S\left(\phi_{L}(x)\right) \nabla \phi_{L}\right)$ should always be from inside to outside of that region. For an easy understanding, we explain the design of the function $S\left(\phi_{L}(x)\right)$ using an example shown in Fig. 1. The green line

\footnotetext{
${ }^{3}$ We note that in the AC segmentation framework, the idea of using labels to perform a multi-phase segmentation has been presented (see for instance Lie et al., 2006). The difference with our work is that this representation has been proposed previously for particular variational energy-models and we present a scheme for any type of PDE-based models.
}

enhances the interface between a high-intensity and a lowintensity label, each representing a different region. Each panel shows the current pixel (enhanced in bold) surrounded by its eight neighbors. The arrows represent the direction of the gradients of the label function which are always from the low intensity label to the high intensity label. Now, if the neighbors of $x$ have higher intensity label values than $\phi_{L}(x)$, it means that the desired level set evolution direction coincides with the gradient direction; so, $S\left(\phi_{L}(x)\right)$ value is taken to be 1 (Fig. 1a). On the other hand, if the neighbors of $x$ have lower intensity label values than $\phi_{L}(x)$, it means that the desired level set evolution direction is opposite to that of the gradient direction of the label function; so, $S\left(\phi_{L}(x)\right)$ value is taken to be -1 (Fig. 1b). Finally, if all the neighbors of $x$ have same label values as that of $x$, it means that the gradient value of the label function is zero, and there is no evolution of the level set for that pixel; so, $S\left(\phi_{L}(x)\right)$ value is taken to be 0 (Fig. 1c). Thus, with the inclusion of this polarity information and the label function, the general formulation of our registration model (10) becomes:

$\frac{\partial u(x, t)}{\partial t}=-S\left(\phi_{L}(x)\right) v\left(\phi_{L}(x+u(x, t), 0)\right) N_{\phi_{L}}$.

To further illustrate about the polarity information, another example of a label function containing three regions $\left(\Omega_{1}, \Omega_{2}, \Omega_{3}\right)$ is presented in Fig. 2. The arrows in Fig. 2a show the orientations of the gradient $\nabla \phi_{L}$ at the interfaces of the label function. Fig. 2b shows the orientations of $\left(S\left(\phi_{L}(x)\right) \nabla \phi_{L}(x)\right)$. In both these figures, arrows in the regions where the polarity information and the gradient are in the opposite direction, are marked in red ${ }^{4}$; if both are in the same direction, they are marked in green.

We now present other important implementation details of the label function. As mentioned in Section 3.2, $\phi_{L}$ value at time $t$ and with the deformation field $u$ is computed using Eq. (9). Note that this computation is done using a nearest neighbor interpolation so that values of the level set function remain fixed $([1, \ldots, n])$ during the registration process. Another point to be mentioned is, since $\phi_{L}$ is not a continuous function across its borders, we convolve it with a Gaussian kernel $G_{\sigma}$ prior to the gradient computation. One of the possible alternatives to Gaussian kernel is using a regularized version of the Heaviside function as in Chan and Vese (2001); however, Chan and Vese (2001) does not treat all level sets equally, giving more weight to the zero level set. Thus, image registration with the proposed multi-label level set representation is more robust if all the level sets are treated equally, which is done with the Gaussian smoothing approach. Further, to stabilize the numerical computation when $\nabla \phi_{L}$ is close to zero, Eq. (12) is modified as follows:

$\frac{\partial u(x, t)}{\partial t}=-S\left(\phi_{L}(x)\right) v\left(\phi_{L}(x+u(x, t), 0)\right) \frac{\nabla\left(G_{\sigma} * \phi_{L}\right)}{\left|\nabla G_{\sigma} * \phi_{L}\right|_{\epsilon}}$.

As mentioned earlier, $\epsilon$ is a small positive constant used for avoiding the singularity. In all our experiments, we set $\epsilon$ to $1 e^{-6}$; we notice that as long as $\epsilon$ is small enough such as $\epsilon<1 e^{-4}$, there are no problems of sensitivity of the method to this parameter.

\section{Driving forces and regularization constraints}

In this section, we first present various types of registration/ segmentation forces that can be used in our registration framework. Secondly, we derive two of the existing atlas registration models (Yezzi's model (Yezzi et al., 2001) and Vemuri's model (Vemuri et al., 2003)) from our general framework, and compare them conceptually with the model that we have proposed in

\footnotetext{
${ }^{4}$ For interpretation of color in Figs. 1-13, the reader is referred to the web version of this article.
} 


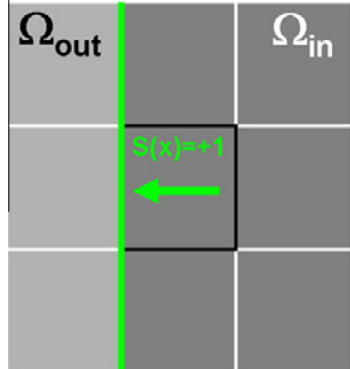

(a)

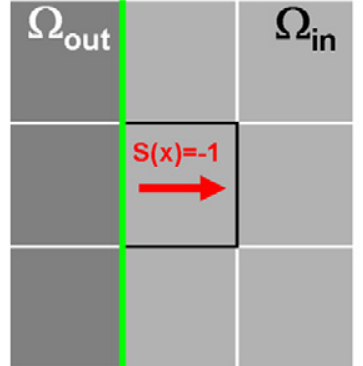

(b)

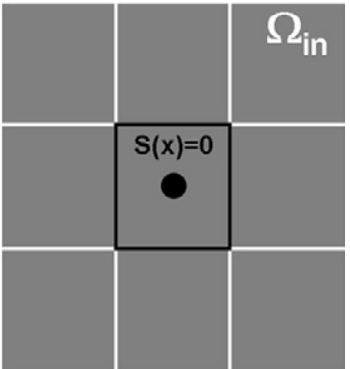

(c)

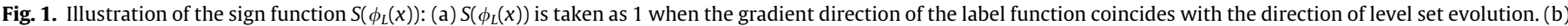

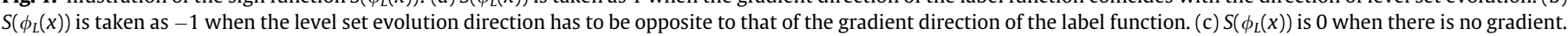

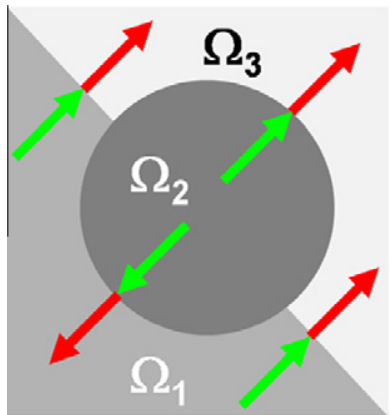

(a)

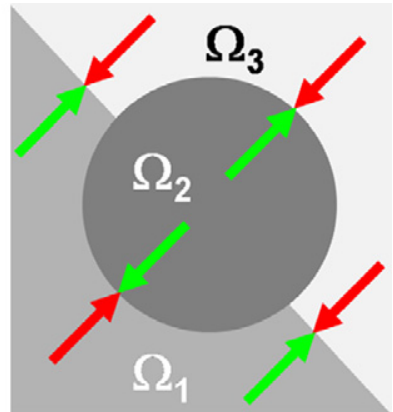

(b)
Fig. 2. Example of a label function with three regions. (a) Orientations of the label function gradients. (b) Orientations of $\left(S\left(\phi_{L}(x)\right) \nabla \phi_{L}(x)\right)$, i.e., with the inclusion of polarity information. Arrows are marked in red when orientations of $\left(\nabla \phi_{L}(x)\right)$ and $\left(S\left(\phi_{L}(x)\right) \nabla \phi_{L}(x)\right)$ are in the opposite directions; arrows are marked in green when both the orientations are same.

Section 3.3. Then, we present the smoothing and the bijectivity constraints that we include in our AC-based registration framework. Then, we describe two types of hierarchical approaches that we use in our implementation. Finally, we present an overview of the whole segmentation approach.

\subsection{Registration/segmentation forces}

One of the main contributions of our registration framework is that it facilitates using various types of AC-based as well as OFbased forces. Thus, this framework can be easily adapted to various specific applications. Fig. 3 classifies the AC-based and OF-based forces according to their effect in a contour matching process. The most used regularization force of the AC framework is the mean

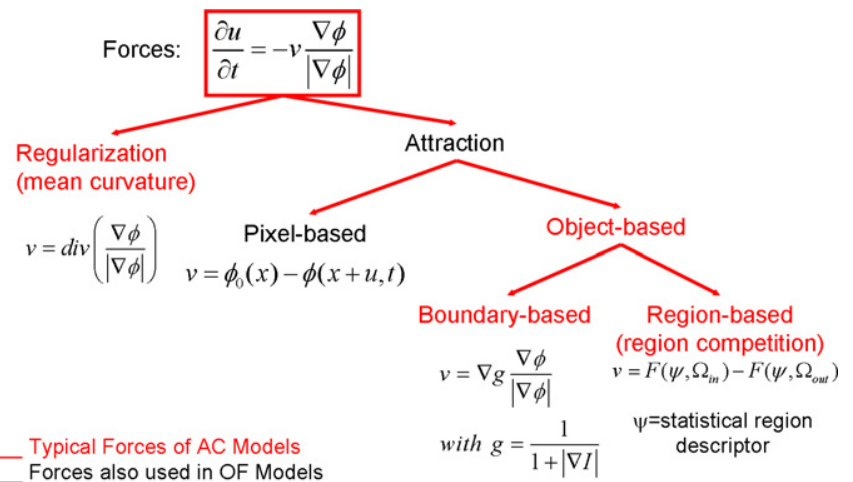

Fig. 3. Classification of the AC-based and OF-based forces according to their effect in a contour matching process. curvature force. This force smoothes the level sets by minimizing their length. They can be applied to any type of contour representation. The pixel-based forces are based on the smallest image feature, the pixel value. They allow the local registration of the whole moving image domain or selected regions. Pixel-based forces are the typical segmentation forces of the OF model. In AC model, these forces are rather used to include intensity or shape prior knowledge in a segmentation process. These forces can match any type of contours (closed or open) and can also be used with any type of representation. However, they are very sensitive to image noise and are limited to recover small deformations. The object-based forces can register image regions. Finally, region-based forces are very efficient forces of the AC framework because they are less sensitive to noise than the boundary-based forces. They can also perform supervised segmentation, i.e., they can use prior knowledge extracted from a reference image. In summary, the current framework allows to choose any of these forces depending on the specific application.

\subsection{Derived atlas-based registration models}

Different models can be derived by simply changing the type of active contour representation $\phi_{G}$, and/or the speed term $v$ that is used to generate the segmentation/registration forces in (10). Note that the possible selections of $v$ are determined/limited by the selection of the level set function $\phi_{G}$. We present here three important special cases of this generalized framework.

(i) If $\phi_{D}$ is used as the level set function in (10), it results in Yezzi's model (Yezzi et al., 2001). With the $\phi_{D}$ representation, only the speed terms related to the object-based forces and the regularization forces can be used, but not the pixelbased forces. As mentioned earlier, $\phi_{D}$ representation can be used only for the two-phase segmentation.

(ii) If $\phi_{I}$ is used as the level set function, it results in the Vemuri's model (Vemuri et al., 2003). With the $\phi_{I}$ representation, only the speed terms related to the pixel-based forces can be used; neither the object-based, nor the regularization forces can be used.

(iii) If the new $\phi_{L}$ representation proposed in Section 3.3 is used, it results in the segmentation model presented in Eq. (13). With this new $\phi_{L}$ representation, speed terms related to any of the three forces can be used. Further, $\phi_{L}$ representation can perform multi-phase segmentation. The above discussion has been summarized in Table 1. Thus, it is clear that the proposed framework generalizes two completely diverse state-of-the-art atlas-based methods, and with our proposed $\phi_{L}$ representation, multi-phase segmentation can be performed besides benefitting from any of the registration forces. 
Table 1

Comparison of our model that uses $\phi_{L}$ representation, with Yezzi et al. (2001) and Vemuri et al. (2003).

\begin{tabular}{llll}
\hline & Yezzi's & Vemuri's & Our model with \\
& model & model & $\phi_{L}$ \\
\hline Level set function & $\phi_{D}$ & $\phi_{I}$ & $\phi_{L}$ \\
Regularization forces & $レ$ & $\times$ & \\
Pixel-based forces & $\times$ & $\checkmark$ & \\
Object-based forces & $\vee$ & $\times$ & \\
Multi-phase & $\times$ & $\vee$ & \\
segmentation & & & \\
\hline
\end{tabular}

\subsection{Regularization constraints}

In order to constrain the output deformation field to be uniform we use two types of regularization constraints: (i) smoothing constraints, (ii) bijectivity constraint; these are described in more detail below. Incorporation of these constraints is required because of the ill posed nature of the non-rigid transformation.

\subsubsection{Smoothing constraints}

Linear diffusion smoothing is the commonly used regularization constraint in non-parametric registration algorithms (see Thirion, 1998; Vemuri et al., 2003). It is applied at the end of each iteration on the current global deformation field. Its PDE corresponds to the well-known heat equation:

$\frac{\partial v(x, t)}{\partial t}=\Delta v(x, t)$

$v(x, t=0)=u_{*}(x)$,

where $u_{*}$ is the solution of Eq. (10) at the point $x$ and $\Delta$ is the Laplacian operator. This technique permits not only to smooth the deformation field, but also to diffuse the contour deformation in a narrow-band around it. The fastest way to perform this diffusion is by filtering. The filter corresponding to the heat equation is the Gaussian filter.

As mentioned in Section 4.1, mean curvature is commonly used in active contour segmentation models for smoothing. Opposed to the linear diffusion, mean curvature approach acts directly on the evolution equation. Its goal is to constrain the evolving contours of the moving image to stay smoothed during their evolution. Hence, in this paper, we apply mean curvature smoothing while using region-based forces, and apply linear diffusion smoothing (through Gaussian filtering) while using pixel-based forces.

\subsubsection{Bijectivity constraint}

In recent years, there are significant developments in the estimation of smooth and invertible transformations with the main goal being the preservation of the topology of objects. In the framework of registration containing large deformations, diffeomorphisms are powerful and mathematically elegant transformations that ensure a one-to-one smooth and continuous mapping with nonsingular Jacobian determinant; they guarantee both invertibility and preservation of topology. Earlier diffeomorphic registration algorithms are based on differential equations-based modeling of "viscous fluid" (Miller et al., 1999; Christensen et al., 2002). Recent diffeomorphic algorithms (Marsland and Twining, 2004; Beg et al., 2005; Avants et al., 2008; Vercauteren et al., 2009) resulted in more accurate estimations, but still, most of these algorithms have a high computational cost.

In a small deformation framework, many registration approaches still use non-diffeomorphic transformations; these algorithms, unlike diffeomorphic methods, are based on simply adding non-parametric displacement fields to an identity transform. However, such approaches do not necessarily preserve the topology, and do not guarantee the bijectivity constraint unless some explicit schemes are implemented like in Thirion (1998) and Christensen and Johnson (2002). Note that our deformation field computation is similar to the "additive Demons iterations scheme" of Thirion's approach (Thirion, 1998) in terms of the way the deformation field is updated, and the type of iterative scheme. Thirion (1998) computes the forward and the backward transformations independently, following the approach suggested by Burr (1981), and modifies these transformations at each iteration to maintain their compatibility.

The backward transformation required in Thirion's approach can be computed in different ways. The simplest method could be to use a second label function defined on the target image, and perform registration with the first image. However, creating a label function on the target image could be cumbersome when segmentation of the structures that are used in the labeled image cannot be obtained using simple segmentation procedures like thresholding. Hence, in order to avoid the segmentation of labeled structures in the target image, we use another method inspired by the Thirions's algorithm (Thirion, 1998). In this approach, the inverse transform is computed by diffusing the object of the target image through the contours of the source label image. This leads to the following equation:

$\frac{\partial u(x, t)}{\partial t}=v\left(\phi_{G}(x+u(x, t), 0)\right) N_{\phi_{G}}$.

Notice that the above equation is identical to (10) except that the label function is not deformed and the driving forces have an opposite sign because they do not have to attract the contour to the target image; rather, it has to diffuse the target object inside the contour. While the above approach, like in Thirion (1998), is good enough for small deformation framework, it may not be an accurate method in case of large deformations. Recently, a diffeomorphic model for the Demons algorithms has been proposed in Vercauteren et al. (2009). We could also probably adapt a similar approach to our framework in order to deal with large deformations; however, this is beyond the scope of this paper.

\subsection{Hierarchical approach to registration forces}

As described in Section 4, our proposed framework facilitates to use multiple registration forces coming from both $O F$ and $A C$ frameworks. Further, the multi-phase AC representation proposed in Section 3.3 enables to select specific regions to be used for each of those registration forces. To benefit fully from these features, we propose to use the registration/segmentation forces in an hierarchical manner, based on their relative global (or local) characteristics. For instance, we know that the pixel-based forces are more local forces compared to the region-based forces. Hence, logically, performing the registration first with the relatively global regionbased forces, and then with the pixel-based forces could avoid converging to a local minimum. In addition, we point out that there are other possible types of hierarchical approaches that can be easily incorporated into our framework, like, hierarchy of structures to be used (Houhou et al., 2005). The hierarchical approach is illustrated in more detail in Section 5.

\subsection{Atlas-based segmentation process}

We now present an overview of the complete segmentation process. The whole atlas-based segmentation process is illustrated in Fig. 4. Active contour-based atlas registration framework described in the preceding sections is used to register the atlas onto the target image. The structures used for driving the registration constitute the label function. These structures are selected based on two criteria: First, they should be closely located (or relevant) 


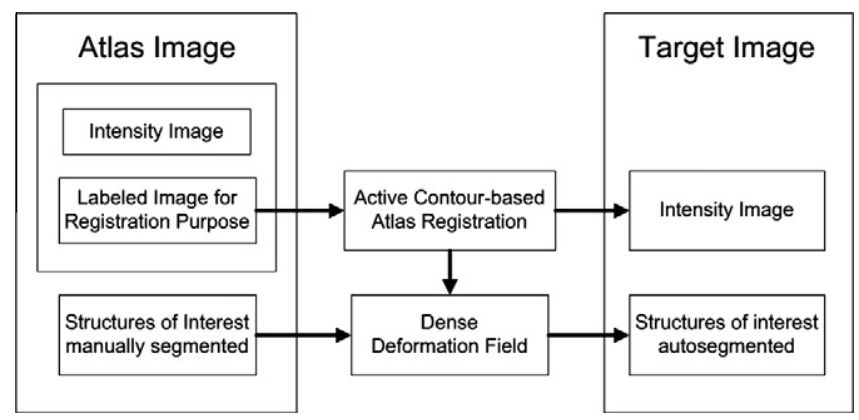

Fig. 4. Block diagram illustrating the proposed atlas segmentation process.

to the structures of interest so that their registration will influence the location of the actual structures to be segmented. Second, structures with distinct characteristics (like edges and region properties) are selected so that they can be accurately delineated by the registration/segmentation forces. Once the dense deformation field matching the atlas to the patient's image is computed, the segmentation process ends up as the classical atlas-based method. The transformation is applied to the manually segmented structures of the atlas image for automatically obtaining their segmentation on the target image.

For some of the applications presented in the next section, we use a region-based force inspired by the unsupervised regionbased segmentation model proposed by Chan and Vese (2001). The following speed function is used for this purpose:

$v=\int_{\Omega_{\text {in }}}\left|I(x)-\mu_{\text {in }}^{\text {prior }}\right|^{2} d x-\int_{\Omega_{\text {out }}}\left|I(x)-\mu_{\text {out }}^{\text {prior }}\right|^{2} d x$,

where $\Omega_{\text {in }}$ is the image area inside the contour and $\Omega_{\text {out }}$ is the image area outside the contour, $\mu^{\text {prior }}$ is the prior mean of a given region extracted from a reference image (the atlas) and $I$ is the intensity function of the image to segment. This force assumes that corresponding regions between the reference and the target images have similar means. ${ }^{5}$

Note that even for multi-phase segmentation with more than two regions, the above energy equation remains same except that the parameters of the label function are interpreted in a slightly different manner; those parameters are not defined anymore with respect to the entire label function. Instead, they are defined for each distinct region of the label function (i.e., based on the value of $\phi_{L}(x)$ ).

With the label function representation and the above meanbased force, the equation of the derived AC-based atlas registration model is:

$\frac{\partial u(x, t)}{\partial t}=-S\left(\phi_{L}(x)\right)\left(\left(I(x)-\mu_{\text {in }}^{\text {prior }}\right)^{2}-\left(I(x)-\mu_{\text {out }}^{\text {prior }}\right)^{2}\right) \frac{\nabla\left(G_{\sigma} * \phi_{L}\right)}{\left|\nabla G_{\sigma} * \phi_{L}\right|_{\epsilon}}$.

The parameters and the numerical approach used for solving the above equation are same as those that are already presented for pixel-based registration (Eq. (13)) except with the following additional important details: Since $\mu^{\text {prior }}$ does not evolve during the registration process, it is computed only once, on the atlas image, at the beginning of the registration. The sign function $S\left(\phi_{L}(x)\right)$ is computed over 8-pixel neighborhood for 2D images, and over 27-voxel neighborhood for 3-D images. The implementation details for $\phi_{L}(x)$ are already presented in Section 3.3.

We would like to mention here regarding the sensitivity of registration towards manual delineations of labels. Notice that the

\footnotetext{
${ }^{5}$ Possible intensity differences between both images can be reduced in a preprocess step by histogram matching.
}

above region-based registration approach requires delineation of selected structures only in the atlas, but not in the target image. It is assumed that the structures of interest are accurately delineated in the atlas. In case of any errors in those manual delineations, deformation field estimated from the region-based registration can get affected accordingly. However, thanks to the pixel-based forces, if they are used in the next level (whenever applicable), since they do not depend on manual delineations, they can indeed correct the previous errors as long as the effect of errors caused in the preceding step are within a retrievable local neighborhood range. The bijectivity constraint can also automatically compensate for small errors in manual segmentations.

Finally, note that although we presented here only the classical mean-based region-forces that use sum of squared intensity differences as the similarity measure for the registration, this framework is not at all limited to only these forces; it can be easily adapted to various other types of metrics, as well as registration forces. For instance, we indeed showed in Duay et al. (2007) how marginal entropies and joint entropy can be used for driving the regionbased registration. Thus, this framework is suitable for even multi-modal registration. It also allows to easily incorporate other measures (like mutual information and its variants) as well, and it could be probably extended to other statistical registration models like (Toews and Wells, 2009). Regarding the incorporation of other types of registration forces, we showed in Houhou et al. (2008) how shape-based forces can also be integrated into our framework

\section{Applications}

In this section, we demonstrate the versatility and potentiality of our framework through a 2D synthetic example and three real clinical applications.

\section{1. $2 D$ synthetic example}

We illustrate here, the behavior of various algorithms, using a 2D synthetic example. Fig. 5 shows the synthetic images used for this purpose. Fig. 5a and b show the target and atlas images respectively. Labeled image of the atlas is shown in Fig. 5c, and is used during the region-based registration. This labeled image, also called as label function, defines four connected regions. Notice that three of these regions (the background, the left and right regions) are consistent between the atlas and the target image, i.e., they have similar mean intensities and texture; on the other hand, the last one (the central region) has a similar mean in both images but the orientation of the texture pattern is different. The target contours are overlayed in red onto the atlas and target images to visualize the initial differences. The arrows in Fig. 5b indicate that in the right region, non-corresponding atlas and target texture patterns having similar intensity distribution are initially superposed.

Fig. 6a shows the results obtained from Vemuri's model (Vemuri et al., 2003). It can be noted that it cannot register correctly in the right and central regions due to the following reasons: (i) The algorithm fails to register global differences because the registration forces that it uses (pixel-based forces) are very local. (ii) Since it has no scheme to prevent the registration of inconsistent regions, it obviously tries to register even the inconsistent texture patterns in the central region.

Fig. 6b shows the results obtained from Yezzi's model (Yezzi et al. (2001)) that uses mean-based forces to register the four regions marked by the labeled image. For this example, while the Yezzi's model has globally registered the regions better than the Vemuri's model, one can see the limitations of using region-based forces alone. Notice from the results that, since the deformation is 


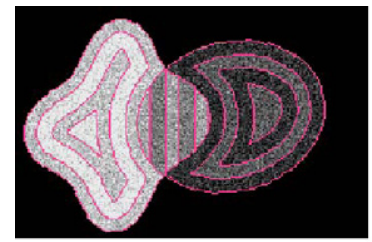

(a)

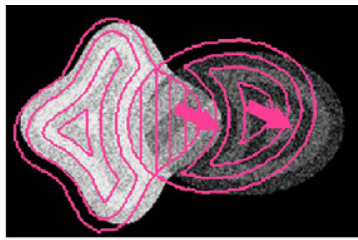

(b)

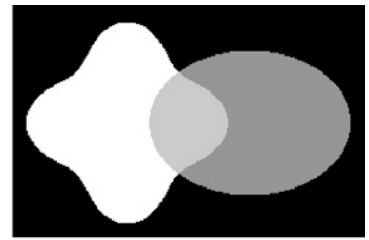

(c)

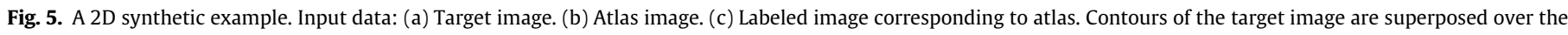
intensity images in order to highlight the initial differences.

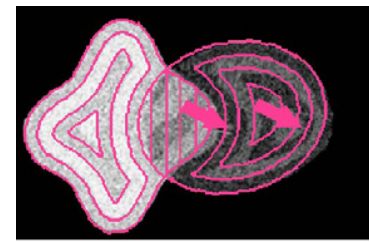

(a) Vemuri's model

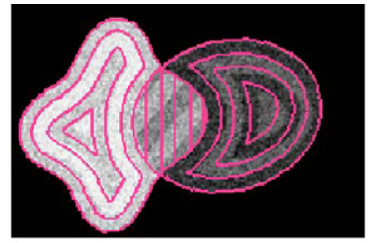

(b) Yezzi's model

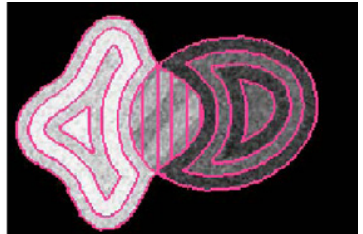

(c) Our model

Fig. 6. Registration results from different methods applied on the synthetic data in Fig. 5 .

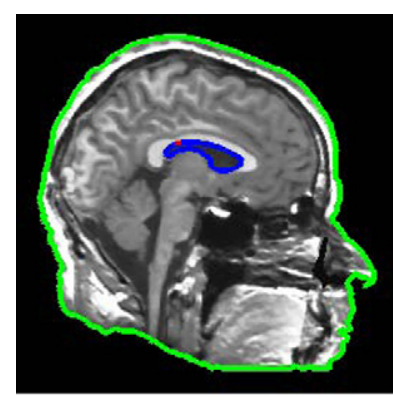

(a)

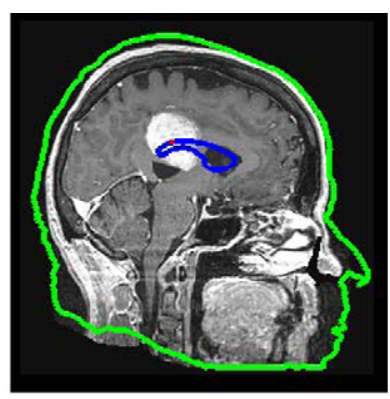

(b)

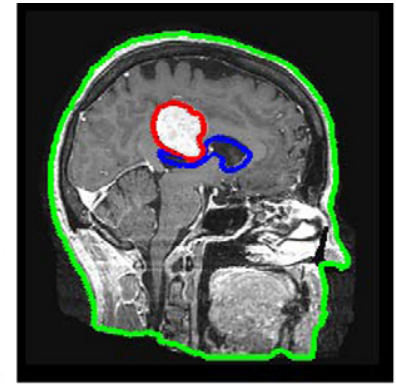

(c)

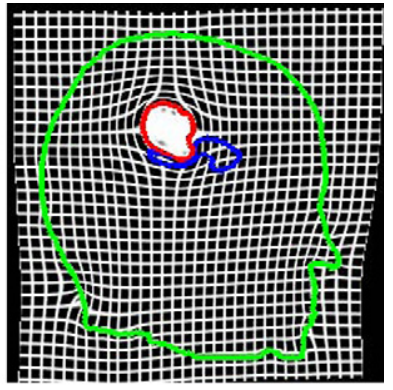

(d)

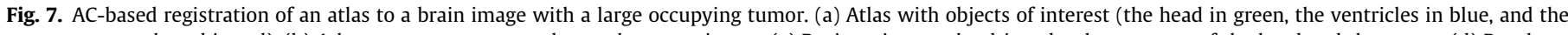

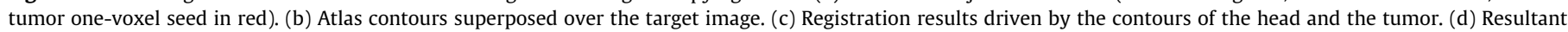
deformation field.

based on only the contours of interest, it has more registration errors in the texture when we are far away from the registered contours. Moreover, we can model only closed contours with the Yezzi's model.

We finally performed the registration with our framework, using the hierarchical approach, and the results are shown in Fig. 6c. In this hierarchical approach, region-based registration is performed followed by the pixel-based registration. Since the texture of the central region is different between the images to be registered, this region is not considered during the pixel-based registration, and thanks to the label function to determine where to compute the pixel-based forces; we just let the texture pattern in the central region to follow the deformation of the region-based contours. We can see from the results on this simple synthetic 2D images that our combined region-based and pixel-based hierarchical model has the potential to give better results than the methods that use only pixel-based forces or, only region-based forces.

\subsection{Atlas registration on a brain MR image with tumor}

The purpose of this first application is to demonstrate with a simple example, how our framework can be adapted to the registration problems where there are inconsistent structures (like tumors) between the atlas and the patient's image. In particular, we show on 2D MR images how our registration framework can be used to grow an inconsistent structure in an atlas.

\subsubsection{Data set}

The images used in this application are obtained from the Surgical Planning Laboratory (SPL) of the Harvard Medical School \& NSG Brain Tumor Database (Kaus et al., 1999; Kikinis et al., 1996). The atlas and the patient's images are shown in Fig. 7a and $b$ respectively. Note that the patient's image contains a structure which not present in the atlas; this inconsistent structure is in fact a tumor that has drastically deformed its surrounding structures. Contours selected in the atlas (the head in green and the ventricles in blue) are overlayed on both the images to visualize the initial differences.

\subsubsection{Our method}

To grow the patient's tumor in the atlas, we use a technique inspired by the tumor growth model we have previously presented in Bach Cuadra et al. (2004). This technique inserts a one-voxel seed (shown by a red point in Fig. 7a) inside the atlas and grows it radially in order to simulate a tumor growth. Here, we propose to model this one-voxel seed as the initial position of an active contour. This way, we do not need a special radial growth scheme anymore. Indeed, the radial evolution of this AC-based seed is 
implicitly defined in the evolution equation of our atlas registration model. Moreover, the active contour is going to segment the tumor of the patient image during the registration process. Thus, the pre-segmentation of the patient tumor is not required unlike with our previous method (Bach Cuadra et al., 2004). We can see that our active contour-based algorithm allows to select the atlas contours that drive its registration. In this application, the atlas registration is driven by the head contour (green contours) and the tumor growth (red contours). We let the ventricles (blue contours) to follow the deformation interpolated from the displacement of the selected contours since, our objective here is also to analyze the influence of the selected contours (green and red) to their surrounding contours (blue). Thus we evolve these contours using the region-based forces described in Section 4.5.

\subsubsection{Results}

Fig. 7c shows the segmentation result obtained after the regionbased registration of the external contour of the head and the tumor. Fig. 7d shows the computed deformation field. We can see that the registration of the selected green and red contours has brought the blue contours closer to their target contours. However, notice that, while the deformation field near the contours of the selected structures is very accurate, it is not so when one is far away from these contours. This behavior is very much expected since we considered here region-based registration with only two structures for driving the registration. In order to obtain a more accurate deformation field throughout the brain volume: (a) more structures should be selected for driving the region-based registration, and (b) as a final step, pixel-based registration can be performed for all those regions that are consistent between the two images (i.e., excluding tumor region). However, in the current application, since the structure to be automatically segmented is only the ventricle, region-based registration driven by the above two structures alone is good enough for the demonstration purpose. This objectbased registration also illustrates the spatial dependance that exists between anatomical structures in the process of registration.

\subsection{Automatic subthalamic nucleus targeting for deep brain stimulation surgery}

In this application, we show how our registration framework can be used to estimate the position of nonvisible brain structures based on surrounding ones. We also perform a quantitative evaluation of six segmentation methods along with the manual segmentations made by two experts (Duay et al., 2008).

Deep brain stimulation of the subthalamic nucleus (STN) has revealed to be the most effective surgical technique for the treatment of Parkinson's disease or other movement disorders (Perlmutter and Mink, 2006). The STN is a very small brain structure not easy to locate due to its bad visibility in common medical imaging modalities such as MR images. Hence, atlas-based segmentation methods are often considered to estimate its position in the patient's image. In our previous works (Sanchez Castro et al., 2005, 2006), we have shown that the position of the STN is spatially influenced by the position of the lateral and third ventricles (Fig. 8a). In this application, study the influence of neighboring structures in the estimation of the STNs location. We also perform a quantitative evaluation of our framework for this application.

\subsubsection{Data set}

In this study, a set of 39 bilaterally implanted parkinsonian patients are considered (78 STNs). Two kinds of images are acquired pre-operatively for each patient: 3D T1-weighted MPRAGE MRI sequence (Siemens Vision, 1.5T, Erlangen, Germany) TR $9.7 \mathrm{~ms}$, TE $4 \mathrm{~ms}$, number of slices/slice thickness: $164 / 1.40 \mathrm{~mm}$, FOV $280 \times 280$, matrix $256 \times 256$, pixel size $1.09 \times 1.09 \mathrm{~mm}$ and few

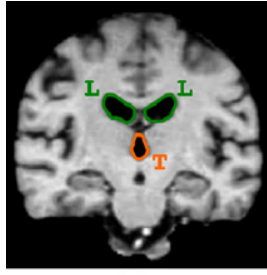

(a)

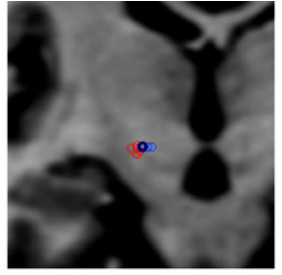

(b)

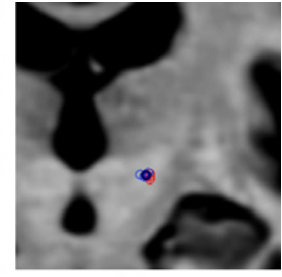

(c)
Fig. 8. Left and right STN atlases (coronal views). (a) Structures selected in the atlas to estimate the position of the STN: lateral ventricles $(L)$ and third ventricle $(T)$. (b and c) Left and right STN ground truth (dark blue) computed from the estimations of two medical experts (Expert 1 in red and Expert 2 in light blue). STN position is represented by a circle of radius $1 \mathrm{~mm}$.

coronal slices of an IR T2-weighted, TR $2560 \mathrm{~ms}$, TE $4 \mathrm{~ms}$, number of slices/slice thickness: $7 / 3 \mathrm{~mm}$, FOV $300 \times 300$, matrix $512 \times$ 512 , pixel size $0.59 \times 0.59 \mathrm{~mm}$. Taking profit from the fact that in some specific patients the STN is visible in MR T2-weighted images a ground truth is constructed from expert's knowledge (Fig. 8b and c) following the protocol described in Sanchez Castro et al. (2005, 2006). Finally, eight patients with clearly visible STNs are selected to take part in this validation process. Among the eight selected patients (16 STNs), the experts have selected the one with the most clearly visible STN as a reference subject, both for the right and left sides.

\subsubsection{Registration methods}

We compare six registration methods along with the manual segmentations made by two independent experts, using the validation scheme proposed in Sanchez Castro et al. (2005, 2006). Out of the six registration methods, three methods perform registration of the whole left and right atlases since, it is not possible with those three methods to select any structures/regions of interest for performing the registration. Those three methods are:

(i) Affine: This is a 12 degrees of freedom (translation, rotation, scaling and shearing) mutual-information-based registration algorithm, based on the work of Maes et al. (1997); this affine registration is also used as a pre-alignment step for the non-rigid registration algorithms that are considered in this application.

(ii) Demons: It is an independent implementation of the intensity-based algorithm (Thirion, 1998).

(iii) BSplines: It is a mutual-information-based free-form deformation algorithm similar to the method proposed in Rueckert et al. (1999). The other three registration methods that we compare are non-rigid registration methods that are used to register only the selected structures of interest (the lateral and third ventricles), and are as follows:

(iv) Segm.-Demons: The Demons registration is applied between the binary masks of the selected structures in the atlas and the patient under study. This algorithm is same as the one that we used in Sanchez Castro et al. (2006) to determine which structures influence the STN position.

(v) RBF: This is a mutual information-based technique in which the deformation that registers the intensity atlas onto the patient's image is modeled with a linear combination of radial basis functions (RBF) using the finite supports that are placed at relevant image point features (Sanchez Castro, 2007).

(vi) Our method $(A C)$ : We perform the registration using our proposed framework; we use the region-based active contour (AC) forces described in Section 4.5. For the FFD-based algorithm, we used the standard BSpline expansion with cubic 
Splines (Sanchez Castro, 2007). To speed-up the optimization process, the algorithm is implemented through an hierarchical multi-scale scheme, both for images and grid of control points. We have tested grids of $4,8,12$ and 16 points in each direction, and a grid of $16 \times 16 \times 16$ points has been considered optimal. No further parameter exploration has been performed for finer grids in order to keep a reasonable computational cost. The parameters used for Affine, Demons, Segm.-Demons and RBF are same as those used in Sanchez Castro (2007).

\subsubsection{Results}

The error estimations are computed as the Euclidean distance from the estimated target given by each method/expert to the ground truth. For evaluating the significant differences, analysis of variance (ANOVA) statistical test is performed. Fig. 9a presents a statistical box plot generated by this test, and Fig. $9 \mathrm{~b}$ presents a multi-comparison test of the means. Fig. 9b shows that the results from Segm.-Demons, our model are statically different from affine registration, RBF and Demons, while they are similar to the experts' inter-variability. Table 2 presents the mean and standard deviation of the errors for all the methods and expert segmentations; it also summarizes the important differences among these methods. The entries in the table are sorted by decreasing mean error. It can be noted that our model using the region-based AC forces gave better results than RBF and Demons whereas, results from BSplines and Segm.-Demons are better than our AC model. However, as pointed out in the table, the advantage of our AC model over Segm.-Demons is that, Segm.-Demons requires prior manual contouring of the structures of interest on the patient's image while our AC model does not require any such manual contouring on the patient's image. The segmentation results from BSplines are only slightly better than our AC model. But, as mentioned in the table, unlike AC model, BSplines method does not facilitate to select the regions of interest for driving the registration and hence, the registration has to be always performed over the whole image. At this point, we want to reemphasize that the purpose of these applications is however not to prove that the proposed framework is the best one for some specific applications. Rather, as mentioned at the beginning of this section, the objective of these applications is to demonstrate the versatility and potential of our generalized framework in adapting to different applications, and validate the results with other state-ofthe-art models. In this application, our framework is using only the region-based AC forces. Unlike other models, it is possible to include additional registration forces in our framework. Future work involves including additional registration forces derived from a prior deformation field model, like the one proposed in Charpiat et al. (2007).

\subsection{Segmentation of head and neck lymph nodes}

Notice that in the preceding clinical applications, we have used only the region-based AC registration forces for our framework. The purpose of this final application is to illustrate the ability and the advantage of our framework to combine multiple registration forces in an hierarchical manner, as described in Section 4.4. In particular, we perform the segmentation of lymph node regions in the 3D Head and Neck (H\&N) CT images using both region-based and pixel-based forces. We also perform a comparison with two
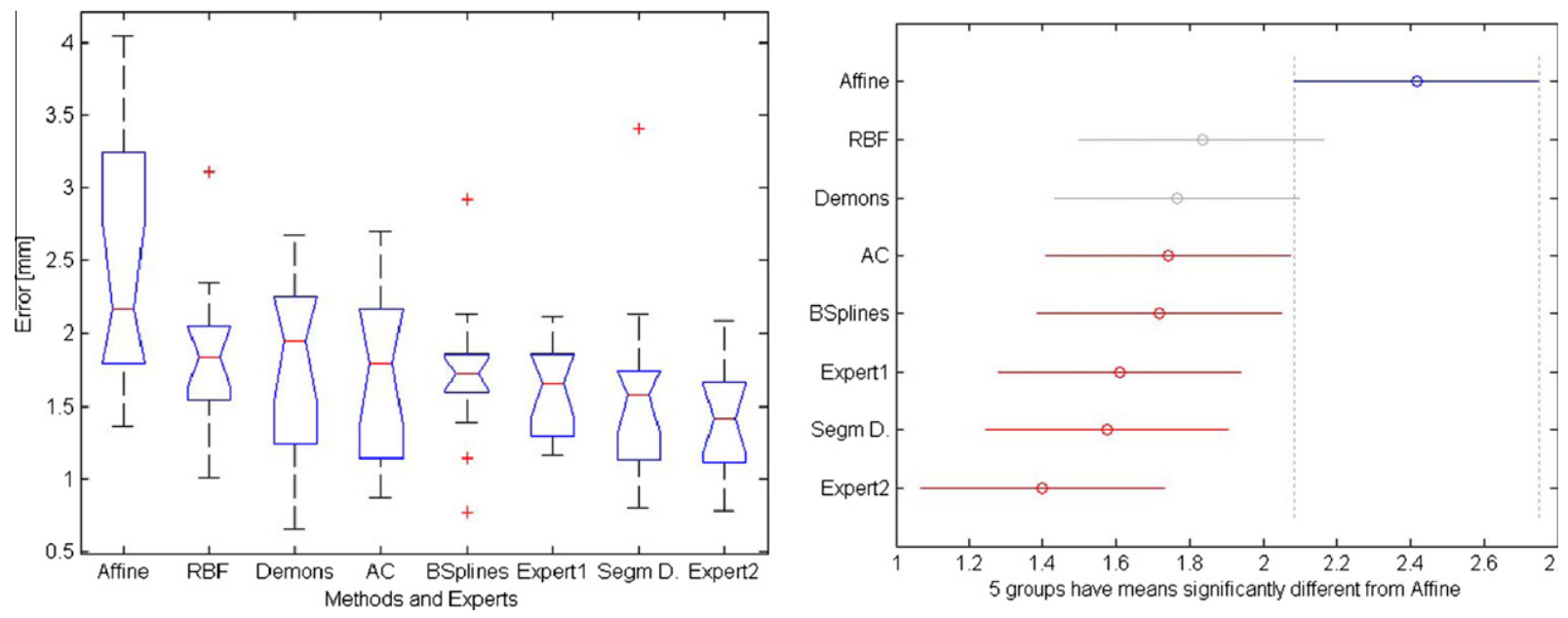

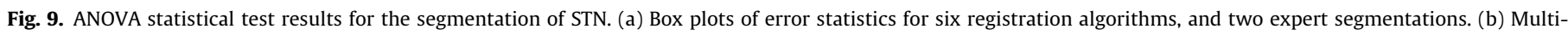
comparison test based on the mean of segmentation errors.

Table 2

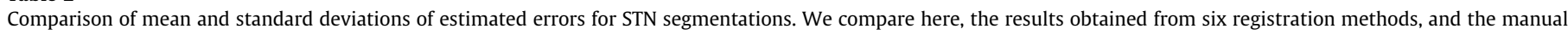
segmentations done by two independent experts. The details of our method are shown in bold.

\begin{tabular}{|c|c|c|c|c|}
\hline S. No. & Method & Estimated error (mm) & Nature of segmentation & Ability to register only selected regions \\
\hline 1 & Affine & $2.42 \pm 0.84$ & Automated & $\times$ \\
\hline 2 & $\mathrm{RBF}$ & $1.83 \pm 0.53$ & Automated & $\boldsymbol{\nu}$ \\
\hline 3 & Demons & $1.77 \pm 0.65$ & Automated & $\times$ \\
\hline 4 & Our Model & $1.74 \pm 0.55$ & Automated & $\boldsymbol{V}$ \\
\hline 5 & BSplines & $1.72 \pm 0.48$ & Automated & $\times$ \\
\hline 6 & Expert 1 & $1.61 \pm 0.29$ & Manual & - \\
\hline 7 & Segm.-Demons & $1.58 \pm 0.71$ & Semi-automated & レ \\
\hline 8 & Expert 2 & $1.40 \pm 0.38$ & Manual & - \\
\hline
\end{tabular}



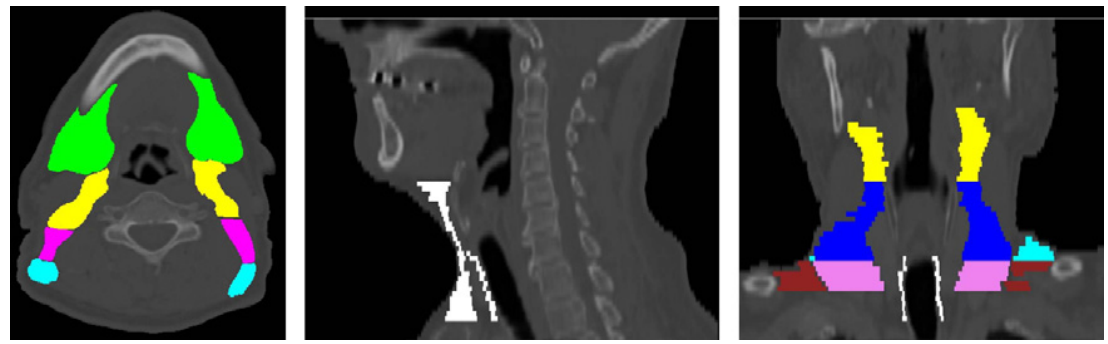

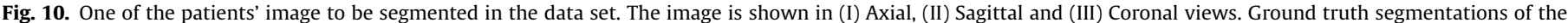
lymph nodes are superposed over the image.

relevant classical models: (i) Vemuri's model (Vemuri et al., 2003) and (ii) Yezzi's model (Yezzi et al., 2001).

Automated segmentation of lymph nodes on 3D CT images is a crucial step for intensity modulated radiotherapy (IMRT) treatment of H\&N cancer. Lymph nodes are constructed volumes that do not have any visibly distinct boundaries with respect to the surrounding structures. Rather, they are defined relative to other visible landmark structures in the CT images, and thus making their segmentation a challenging task. Fig. 10 shows the manually delineated ground truth segmentations of lymph node levels: IB-Left, IB-Right, IIA-Left, IIA-Right, III-Left, III-Right, IV-Left, IV-Right, VALeft, VA-Right, VB-Left, VB-Right, and VI. The ground truth segmentations are performed by a medical expert, under the supervision of a radiation oncologist. In Gorthi et al. (2009), we used a preliminary version of the current framework with the main focus being the specific H\&N lymph nodes segmentation problem. We now perform a detailed evaluation with the current framework, and also study the effect of combining various registration forces in an hierarchical manner for this application.

\subsubsection{Data set}

The data set used for this application contains the H\&N CT images of 13 patients, acquired at Divisions of Radiotherapy, Geneva University Hospital (HUG), during the routine clinical practice. The resolution of $C T$ images in $X$ and $Y$ directions is varying in the range of $0.45-0.94 \mathrm{~mm}$. The typical resolution in the $Z$ direction is $3 \mathrm{~mm}$. The size of each axial slice is $512 \times 512$ pixels. The Field of View (FOV) was not same for all the images, and hence, the images are cropped during the preprocessing to contain the same FOV. The number of slices for the data set after cropping is varying in the range of $61-73$.

\subsubsection{Registration methods}

We compare here three registration models: In the first case, we use our framework with only the pixel-based forces for performing the registration; thus, this is equivalent to using the Vemuri's model (Vemuri et al., 2003). In the second case, we use our framework with only the region-based forces for performing the registration; thus, this is equivalent to using Yezzi's model (Yezzi et al., 2001). In the final case, we consider both the region-based and pixelbased forces in an hierarchical manner. Notice that this hierarchical approach became possible because of the proposed framework. As mentioned earlier, since region-based forces are more global than the pixel-based forces, during the hierarchical registration, we first use the region-based forces, and then followed by the pixel-based forces.

The structures that have been selected for driving the regionbased registration are: (i) external contours of the $\mathrm{H} \& \mathrm{~N}$, (ii) bones, and (iii) trachea, thus forming four distinct labels (including the background). Fig. 11 shows these labels for one of the patients. Notice that all the above selected structures have unique intensity characteristics (i.e., mean values of intensities inside and outside the selected structures), and they also influence the location of the lymph nodes to be segmented. During the region-based registration, curvature-based regularization is also used on the resulting contour. Gaussian smoothing with a sigma of $1.5 \mathrm{~mm}$ is used in the region-based registration whereas a sigma of $3.5 \mathrm{~mm}$ is used in the pixel-based registration.

There can be huge anatomical variations in the $H \& N$ region among different patients. In addition to the registration method used, similarity/closeness of the atlas to the patient's image to be segmented also plays an important role in the segmentation accuracy. If the atlas is more similar to the patient to be segmented, better will be the segmentation results. Hence, unlike in the other two applications, a single arbitrarily selected image is not used as atlas for all the images in the data set; rather, for each image to be segmented, an atlas is adaptively selected from the rest of the images in the data set, based on the Mean Square Error (MSE) metric. For this purpose, each image to be segmented is first affinely registered to the other images in the data set, using leaveone-out strategy; then MSE value is computed over the entire image region for each pair of affinely registered images; the image that has given the least MSE is chosen as the atlas for that patient's image to be segmented.
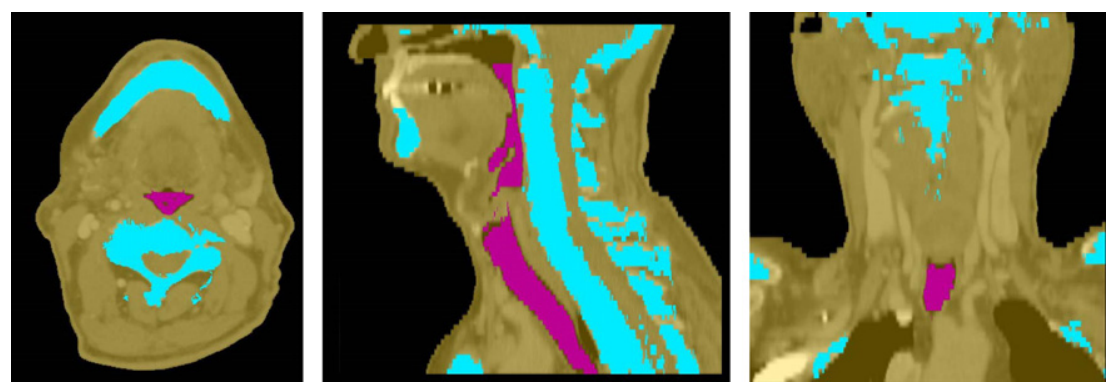

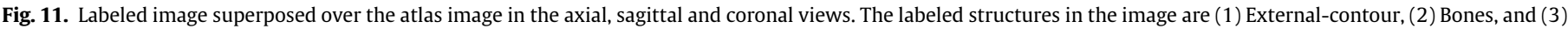
Trachea. This image is used for driving the region-based registration. 
Notice that use of statistical similarity measures compared to MSE metric could probably reduce the sensitivity to atlas selection. However, the type of impact of the reduced-sensitivity on the accuracy of segmentation can largely depend on the nature of structures to be segmented. For instance, when the structures to be segmented are thin or soft tissue structures with slowly varying intensities, statistical similarity measures could unfortunately be very insensitive in accurately registering those regions (i.e., negative impact of decreased-sensitivity); thus, for such structures, they are not preferable over MSE metric. On the contrary, for example, if structures are not thin and containing some artifacts, statistical measures could probably result in more accurate as well as robust registration results than MSE metric. Since lymph nodes are soft tissue structures, we preferred MSE for this application.

\subsubsection{Results}

Fig. 10 shows one of the patients' image to be segmented. Ground truth segmentations of lymph nodes for that patient are superposed over the same image. Fig. 11 shows the atlas image that was selected for the above patient's image, based on MSE criteria. Labeled image used for driving the region-based (first level) registration is superposed over the same image. In order to qualitatively illustrate the advantage of the proposed hierarchical approach, segmentation results from the three methods in one of the axial slices, along with its ground truth, are presented in Fig. 12; the names of the lymph nodes are labeled in the sub-figure that shows ground truth segmentations. We can see the advantage of the hierarchical approach, particularly for lymph nodes IB and VA. Notice that, in this slice, these are the lymph nodes that are close to the structures considered during region-based registration, and thus, profiting better from both Yezzi's model and Vemuri's model. Hence, with reference to the ground truth, we can see that these results are better compared to using only one of the two methods.

The segmentation results are quantitatively compared using two statistical metrics: (i) Sensitivity, (ii) Dice Similarity Measure (DSM), and a geometrical metric: (i) Mean Hausdorff Distance (HD). Sensitivity metric gives a measure of true positive fraction; higher the sensitivity (ideal value $=1$ ), better the segmentation accuracy. DSM gives a measure of overlap between the ground truth segmentation and automated segmentation; higher DSM value (ideal value $=1$ ) implies a better overlap between the ground truth and automated segmentations. Mean HD gives a measure of mean value of geometrical deviation (in $\mathrm{mm}$ ) between the contours of the ground truth and automated segmentations. Lower the mean HD (ideal value $=0 \mathrm{~mm}$ ), better the automated segmentations. Fig. 13 shows the box plots of Sensitivity, DSM and mean HD when different types of registration forces are used. The corresponding mean and standard deviation values are summarized in Table 3. It can be noted from these results that for all the three measures, our hierarchical registration approach combining region-based and pixel-based forces gave better results than the Vemuri's model (using only pixel-based forces) and the Yezzi's model (using only region-based forces).

We also performed tests to evaluate the statistical significance of differences between the methods. We used Wilcoxon signed-rank test (Wilcoxon, 1945) for this purpose; this is a non-parametric test procedure for the analysis of matched-paired data. Note that Wilcoxon signed-rank test can be seen as non-parametric alternative to paired-student test since it does not make any assumptions

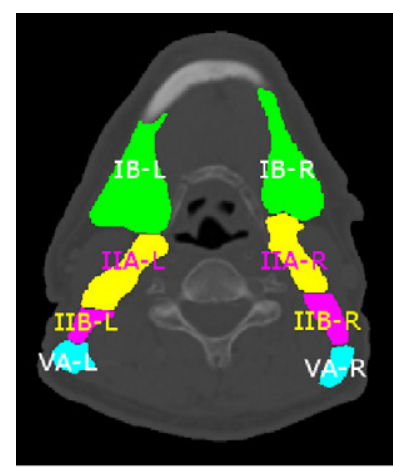

(a) Ground truth

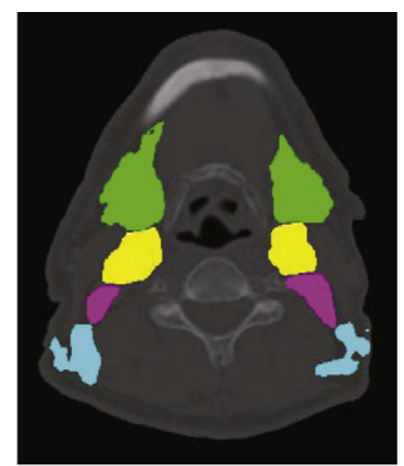

(b) Vemuri's model

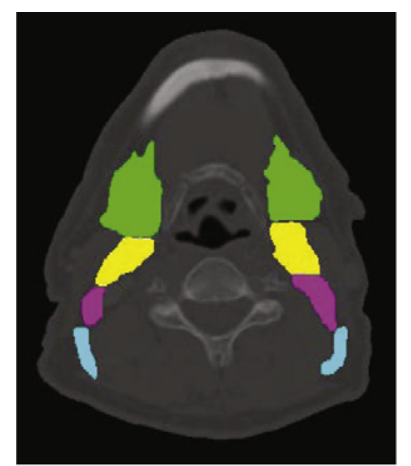

(c) Yezzi's model

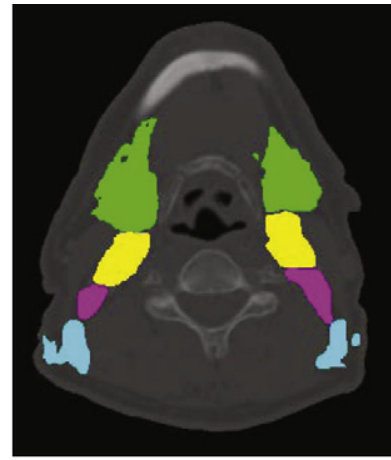

(d) Hierarchical model

Fig. 12. Ground truth and automated segmentations obtained from the three approaches in one of the axial slices.
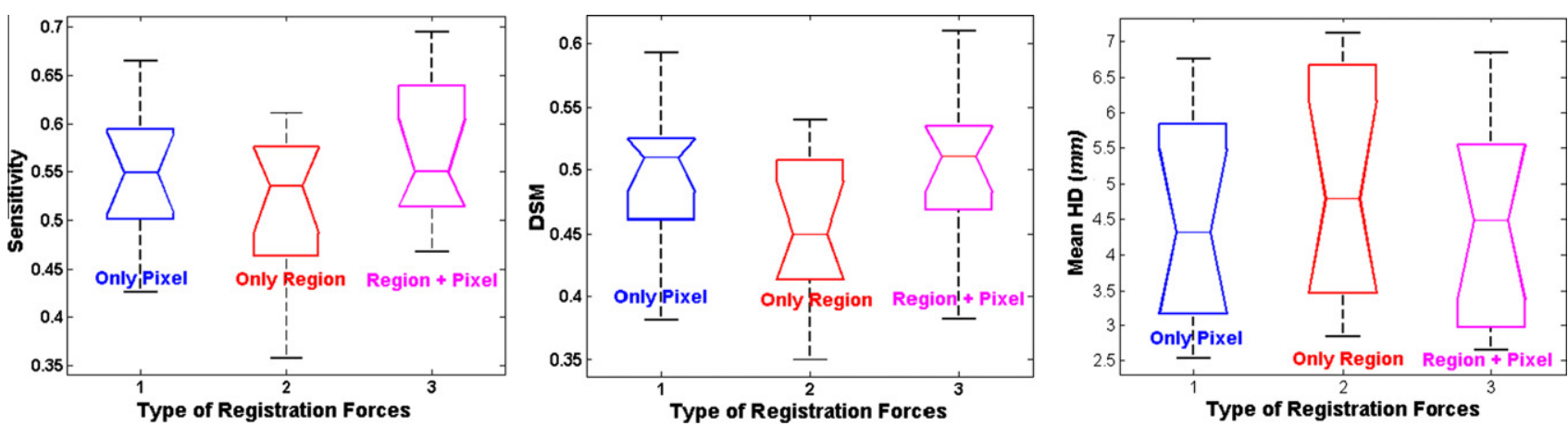

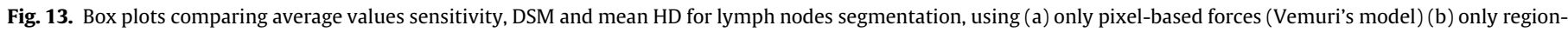
based forces (Yezzi's model) and (c) our hierarchical model. 
Table 3

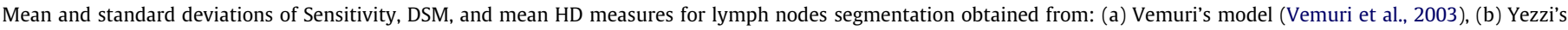
model (Yezzi et al., 2001), and (c) our hierarchical model.

\begin{tabular}{|c|c|c|c|c|}
\hline Method & Type of forces & Sensitivity & DSM & Mean HD $(\mathrm{mm})$ \\
\hline Vemuri's model & Only pixel & $0.550 \pm 0.066$ & $0.494 \pm 0.056$ & $4.507 \pm 1.412$ \\
\hline Yezzi's model & Only region & $0.518 \pm 0.072$ & $0.451 \pm 0.062$ & $5.043 \pm 1.669$ \\
\hline Our model & Region + pixel & $\mathbf{0 . 5 7 4} \pm \mathbf{0 . 0 7 0}$ & $0.503 \pm 0.057$ & $4.449 \pm 1.418$ \\
\hline
\end{tabular}

regarding the distributions of the data population. We performed this test on DSM statistics, for each permutation of pair of methods chosen from the three methods, with the alternative hypothesis being: "Segmentation results from the first method are statistically better (greater) than the second method." Based on these results, the following conclusions are drawn for this application at 0.05 significance level: (i) Segmentation results from Vemuri's model are better than Yezzi's model (with a $p$-value of 0.0004). (ii) Segmentation results from our hierarchical model are better than Vemuri's model (with a $p$-value of 0.009 ) as well as Yezzi's model (with a $p$-value of 0.0001 ).

\section{Conclusions}

The main contribution of this paper is a new and original framework that encompasses many existing registration methods. We have presented how active contour (AC) based registration model of Yezzi et al. (2001), and pixel-based registration model of Vemuri et al. (2003) can be derived from the proposed framework. We have also proposed a new label function for performing multiphase AC-based registration. The advantage of the proposed label function is that it can distinguish any number of regions with a single function. Our proposed framework allows to select the regions/ structures to be used for driving the registration process. This way, the atlas can be registered on target images using the objects that are consistent between both the images. Moreover, registering only the structures that are relevant to estimate the position of the objects of interest, limits the possibilities of mis-registration and also reduces the computational time. We have proposed to use multiple registration forces in an hierarchical manner, based on their relative global characteristics so that convergence to a local minimum can be avoided besides benefitting from multiple registration forces.

We have demonstrated the versatility of the proposed framework through three diverse clinical applications. In these applications, we mainly used the classical region-based forces (with mean square error (MSE) metric on intensities), and pixel-based forces. The proposed framework facilitates even combining various other forces like shape-based forces. Further, besides MSE metric, it also allows to use information theoretic similarity measures like mutual information and its variants; hence, this framework can be adapted even for multi-modal registration. In future work, we would like to explore combining additional forces as well as using other similarity metrics. It would be also interesting to modify the currently used bijectivity constraints with a diffeomorphic approach.

\section{Acknowledgments}

This work is supported by the Swiss National Science Foundation under Grants: 205320-101621, 3252B0-107873, and 205321-124797, the Center for Biomedical Imaging (CIBM) of the Geneva - Lausanne Universities and the EPFL, and the foundations Leenaards and Louis-Jeantet. We thank Ulrike Schick for ground truth segmentations of the head and neck lymph nodes. We thank Prof. R. Meuli and Prof. P. Maeder from Department of Radiology of the Centre Hospitalier Universitaire Vaudois (CHUV), Lausanne, and the Surgical Planning Laboratory (SPL) of the Harvard Medical School, and NSG Brain Tumor Database for the brain MR images. This work is benefited from the use of the Insight Segmentation and Registration Toolkit (Ibanez et al., 2005).

\section{References}

Amiaz, T., Kiryati, N., 2006. Piecewise-smooth dense optical flow via level sets. International Journal of Computer Vision 68, 111-124

An, J.H., Chen, Y., Huang, F., Wilson, D., Geiser, E., 2005. A variational PDE based level set method for a simultaneous segmentation and non-rigid registration. In: Proceedings of the Medical Image Computing and Computer-Assisted Intervention, MICCAI, pp. 286-293.

Avants, B., Epstein, C., Grossman, M., Gee, J., 2008. Symmetric diffeomorphic image registration with cross-correlation: evaluating automated labeling of elderly and neurodegenerative brain. Medical Image Analysis 12, 26-41.

Bach Cuadra, M., Pollo, C., Bardera, A., Cuisenaire, O., Villemure, J., Thiran, J.P., 2004. Atlas-based segmentation of pathological MR brain images using a model of lesion growth. IEEE Transactions on Medical Imaging 23, 1301-1314.

Bajcsy, R., Kovacic, S., 1989. Multiresolution elastic matching. Computer Vision, Graphics, and Image Processing 46, 1-21.

Barron, J.L., Fleet, D.J., Beauchemin, S.S., 1994. Performance of optical flow techniques. International Journal of Computer Vision 12, 43-77.

Beg, M., Miller, M., Trouvé, A., Younes, L., 2005. Computing large deformation metric mappings via geodesic flows of diffeomorphisms. International Journal of Computer Vision 61, 139-157.

Bertalḿ́, M., Sapiro, G., Randall, G., 2000. Morphing active contours. IEEE Transactions on Pattern Analysis and Machine Intelligence 22, 733-737.

Burr, D.J., 1981. A dynamic model for image registration. Computer Graphics and Image Processing 15, 102-112.

Chan, T.F., Vese, L.A., 2001. Active contours without edges. IEEE Transactions on Image Processing 10, 266-277.

Charpiat, G., Faugeras, O., Keriven, R., 2007. Shape statistics for image segmentation with prior. In: Proceedings of the IEEE Computer Society Conference on Computer Vision and Pattern Recognition (CVPR), pp. 1-6.

Chen, Y., Tagare, H.D., Thiruvenkadam, S., Huang, F., Wilson, D., Gopinath, K.S. Briggs, R.W., Geiser, E.A., 2002. Using prior shapes in geometric active contours in a variational framework. International Journal of Computer Vision 50, 315328.

Christensen, G., Johnson, H., 2002. Consistent image registration. IEEE Transactions on Medical Imaging 20, 568-582.

Christensen, G., Rabbitt, R., Miller, M., 2002. Deformable templates using large deformation kinematics. IEEE Transactions on Image Processing 5, 1435-1447.

Christensen, G.E., Rabbitt, R.D., Miller, M.I., 1994. 3D brain mapping using a deformable neuroanatomy. Physics in Medicine and Biology 39, 609-618.

Droske, M., Ring, W., Rumpf, M., 2009. Mumford-Shah based registration: a comparison of a level set and a phase field approach. Computing and Visualization in Science 12, 101-114.

Duay, V., Bresson, X., Sanchez Castro, F.J., Pollo, C., Bach Cuadra, M., Thiran, J.P., 2008. An active contour-based atlas registration model for automatic subthalamic nucleus targeting on MRI: method and validation. In: Medical Image Computing and Computer-Assisted Intervention, MICCAI, pp. 980-988.

Duay, V., D'Haese, P.F., Li, R., Dawant, B., 2004. Non-rigid registration algorithm with spatially varying stiffness properties. In: IEEE International Symposium on Biomedical Imaging: Nano to Macro, pp. 408-411.

Duay, V., Luti, S., Menegaz, G., Thiran, J., 2007. Active contours and information theory for supervised segmentation on scalar images. In: 15th European Signal Processing Conference, pp. 1769-1773.

Gorthi, S., Duay, V., Houhou, N., Bach Cuadra, M., Schick, U., Becker, M., Allal, A.S., Thiran, J.P., 2009. Segmentation of head and neck lymph node regions for radiotherapy planning using active contour-based atlas registration. IEEE Journal on Selected Topics in Signal Processing 3, 135-147.

Houhou, N., Duay, V., Allal, A.S., Thiran, J.P., 2005. Medical images registration with a hierarchical atlas. In: 13th European Signal Processing Conference.

Houhou, N., Lemkaddem, A., Duay, V., Allal, A., Thiran, J., 2008. Shape prior based on statistical map for active contour segmentation. In: 15th IEEE International Conference on Image Processing, pp. 2284-2287.

Hui, E., Mohamed, S., Salama, M., Fenster, A., 2009. Prostate TRUS image regionbased feature extraction and evaluation. Image Analysis and Recognition, vol. 5627. Springer, Berlin/Heidelberg, pp. 759-771. 
Ibanez, L., Schroeder, W., Ng, L., Cates, J., 2005. The ITK Software Guide, second ed. Kitware, Inc. ISBN:1-930934-15-7.

Kaus, M., Warfield, S., Nabavi, A., Chatzidakis, E., Black, P., Jolesz, F., Kikinis, R., 1999. Segmentation of meningiomas and low grade gliomas in MRI. In: Medical Image Computing and Computer-Assisted Intervention, vol. 1679, pp. 1-10.

Kikinis, R., Shenton, M.E., Losifescu, D.V., McCarley, R.W., Saiviroonporn, P., Hokama, H.H., Robatino, A., Metcalf, D., Wible, C.G., Portas, C.M., Donnino, R.M., Jolesz, F.A., 1996. A digital brain atlas for surgical planning, model-driven segmentation, and teaching. IEEE Transactions on Visualization and Computer Graphics 2, 232-241.

Lie, J., Lysaker, M., Tai, X., 2006. A variant of the level set method and applications to image segmentation. Mathematics of Computation 75, 1155-1174.

Maes, F., Collignon, A., Vandermeulen, D., Marchal, G., Suetens, P., 1997. Multimodality image registration by maximization of mutual information. IEEE Transactions on Medical Imaging 16, 187-198.

Marsland, S., Twining, C., 2004. Constructing diffeomorphic representations for the groupwise analysis of nonrigid registrations of medical images. IEEE Transactions on Medical Imaging 23, 1006-1020.

Miller, M., Joshi, S., Christensen, G., 1999. Large deformation fluid diffeomorphisms for landmark and image matching. Brain Warping, 115-131.

Moelich, M., Chan, T., 2003. Joint segmentation and registration using logic models. Technical Report, UCLA CAM Report.

Osher, S., Sethian, J., 1988. Fronts propagating with curvature-dependent speed algorithms based on Hamilton-Jacobi formulations. Journal of Computational Physics 79, 12-49.

Paragios, N., Deriche, R., 2002. Geodesic active regions and level set methods for supervised texture segmentation. International Journal of Computer Vision 46, 223-247.

Paragios, N., Rousson, M., Ramesh, V., 2002. Matching distance functions: a shapeto-area variational approach for global-to-local registration. In: European Conference on Computer Vision (ECCV), pp. 775-789.

Paragios, N., Rousson, M., Ramesh, V., 2003. Non-rigid registration using distance functions. Computer Vision and Image Understanding 89, 142-165.

Perlmutter, J.S., Mink, J.W., 2006. Deep brain stimulation. Annual Review of Neuroscience 29, 229-257.

Rohlfing, T., Brandt, R., Menzel, R., Russakoff, D.B., Maurer Jr., C.R., 2005. Quo vadis, atlas-based segmentation? The Handbook of Medical Image Analysis, vol. III. Springer, pp. 435-486 (Chapter 11).
Rueckert, D., Sonoda, L.I., Hayes, C., Hill, D.L., Leach, M.O., Hawkes, D.J., 1999. Nonrigid registration using free-form deformations: application to breast MR images. IEEE Transactions on Medical Imaging 18, 712-721.

Sanchez Castro, F., Pollo, C., Meuli, R., Maeder, P., Cuisenaire, O., Bach Cuadra, M. Villemure, J.G., Thiran, J.P., 2006. A cross validation study of deep brain stimulation targeting: from experts to atlas-based, segmentation-based and automatic registration algorithms. IEEE Transactions on Medical Imaging 25 $1440-1450$.

Sanchez Castro, F., Pollo, C., Villemure, J., Thiran, J., 2005. Automatic subthalamic nucleus targeting for deep brain stimulation. A validation study. In: Proceedings of the 19th International Congress and Exhibition in Computer Assisted Radiology and Surgery.

Sanchez Castro, F.J., 2007. Nonrigid medical image registration: algorithms, validation and applications. Ph.D. thesis, EPFL.

Thirion, J.P., 1998. Image matching as a diffusion process: an analogy with Maxwell's Demons. Medical Image Analysis 2, 243-260.

Toews, M., Wells, W., 2009. Bayesian registration via local image regions: information, selection and marginalization. In: Information Processing in Medical Imaging, pp. 435-446.

Unal, G., Slabaugh, G., 2005. Coupled pdes for non-rigid registration and segmentation. In: IEEE Computer Society Conference on Computer Vision and Pattern Recognition (CVPR), pp. 168-175.

Vemuri, B.C., Ye, J., Chen, Y., Leonard, C.M., 2003. Image registration via level-set motion: applications to atlas-based segmentation. Medical Image Analysis 7, 1-20.

Vercauteren, T., Pennec, X., Perchant, A., Ayache, N., 2009. Diffeomorphic Demons: efficient non-parametric image registration. NeuroImage 45, S61-S72.

Vese, L.A., Chan, T.F., 2002. A multiphase level set framework for image segmentation using the Mumford and Shah model. International Journal of Computer Vision 50, 271-293.

Wilcoxon, F., 1945. Individual comparisons by ranking methods. Biometrics Bulletin $1,80-83$.

Yezzi, A., Zollei, L., Kapur, T., 2001. A variational framework for joint segmentation and registration. Proceedings of the IEEE Workshop on Mathematical Methods in Biomedical Image Analysis (CVPR-MMBIA), 44-49.

Young, Y.N., Levy, D., 2005. Registration-based morphing of active contours for segmentation of CT scans. Mathematical Biosciences and Engineering 2,79-96.

Zhao, H.K., Chan, T., Merriman, B., Osher, S., 1996. A variational level set approach to multiphase motion. Journal of Computational Physics 127, 179-195. 